\title{
Energy dependent track structure parametrisations for protons and carbon ions based on nanometric simulations ${ }^{\star}$
}

\author{
Frauke Alexander ${ }^{1, a}$, Carmen Villagrasa ${ }^{2}$, Hans Rabus ${ }^{3}$, and Jan J. Wilkens ${ }^{1}$ \\ 1 Department of Radiation Oncology, Technische Universität München, Klinikum rechts der Isar, Ismaninger Straße 22, \\ 81675 München, Germany \\ 2 Institut de Radioprotection et Sûreté Nucléaire (IRSN), 92262 Fontenay-aux-Roses, France \\ 3 Physikalisch-Technische Bundesanstalt (PTB), 38116 Braunschweig, Germany
}

Received 31 March 2015 / Received in final form 13 July 2015

Published online 17 September 2015 - (C) EDP Sciences, Società Italiana di Fisica, Springer-Verlag 2015

\begin{abstract}
The BioQuaRT project within the European Metrology Research Programme aims at correlating ion track structure characteristics with the biological effects of radiation and develops measurement and simulation techniques for determining ion track structure on different length scales from about $2 \mathrm{~nm}$ to about $10 \mu \mathrm{m}$. Within this framework, we investigate methods to translate track-structure quantities derived on a nanometre scale to macroscopic dimensions. Input data sets were generated by simulations of ion tracks of protons and carbon ions in liquid water using the Geant 4 Monte Carlo toolkit with the Geant4-DNA processes. Based on the energy transfer points - recorded with nanometre resolution we investigated parametrisations of overall properties of ion track structure. Three different track structure parametrisations have been developed using the distances to the 10 next neighbouring ionisations, the radial energy distribution and ionisation cluster size distributions. These parametrisations of nanometric track structure build a basis for deriving biologically relevant mean values which are essential in the clinical situation where each voxel is exposed to a mixed radiation field.
\end{abstract}

\section{Introduction}

High energy ion beams are progressively used for the treatment of cancer patients, as the tumor can be targeted with higher accuracy and organs at risk can be spared more effectively compared to conventional photon therapy [1]. However it is still a current topic of interest to find a reliable way to estimate the biological effectiveness of ion beams. Benefiting from a multi-scale approach, the BioQuaRT ${ }^{1}$ project $[2,3]$ aims at creating a new dosimetric quantity to define the local radiation quality, which strongly influences the biological response. In the long run, this quantity should be integrated into clinical treatment planning systems which simulate and optimise the treatment for each individual patient. Within this framework we studied the spatial distribution of energy depositions on the nanometer scale. Several descriptors of the spatial structure of energy depositions can be defined and are candidates for the new dosimetric quantity mentioned above. With regard to a future clinical use of nanometric track structure properties in clinical applications it is important to develop methods that enable a fast calculation of

\footnotetext{
* Contribution to the Topical Issue "COST Action NanoIBCT: Nano-scale Processes Behind Ion-Beam Cancer Therapy", edited by Andrey Solov'yov, Nigel Mason, Gustavo García, Eugene Surdutovich.

a e-mail: Frauke.Alexander@tum.de

${ }^{1}$ http://www.ptb.de/emrp/bioquart.html
}

the track structure properties describing the relevant features of the spatial distribution. We therefore present here three parametrisations which can be used to extract track structure properties for each desired energy in a clinical energy range without the need to perform a full nanometric Monte Carlo simulation for each patient.

These parametrisations may be useful for treatment planning, where the three-dimensional patient geometry is represented by voxels with a size of $\approx 1 \mathrm{~mm}^{3}$. These voxels are exposed to a mixed radiation field where several particle types with different energies hit a voxel from different directions. The methods presented in this paper can be the basis for the development of a data base of parametrisations that enables a fast computation of the relevant track structure parameters for different particle types and energies present in a mixed radiation field. This is crucial as treatment planning typically involves the evaluation of various possible treatment plans, and it would not be feasible to perform a full track structure simulation with nanometre resolution for each particle type and energy present in each voxel.

\section{Monte Carlo simulations of track structure with nanometre resolution}

The underlying data have been produced using the Geant4 Monte Carlo toolkit [4] (version 9.6) and, in particular, 
the Geant4-DNA processes $[5,6]$ that are included in the Low Energy Package. The models in Geant4-DNA allow simulating the transport in liquid water of electrons, protons, helium particles and heavier ions like carbon ions. The simulated processes are ionisation, excitation and charge change for protons and helium particles and also include elastic scattering, vibrational excitation and attachment for electrons down to the electron volt scale. For other ions, as carbon, the energy loss is mainly due to the ionisation process. In Geant4-DNA the corresponding cross-sections were calculated from the ones of protons using an speed scaling procedure. The energy loss through charge exchange is not explicitly taken into account but, the use of an effective charge of the ion takes into account the screening effect of the shell electrons of the incident particle depending on the ion speed and the media. Thus, using this approximation, we believe that the simulation for carbon ions is accurate enough for the methodological approach presented in this paper. The Geant4-DNA processes are simulated in a step-by-step basis (not condense history approximation) and the low energy cut for the electron transport allows simulating the energy deposition with nanometric resolution, that is what is currently known as a track structure simulation. In particular, a cut of $7.4 \mathrm{eV}$ for the electron transport was used in this work (validity limit for the elastic scattering). For more details, the reader is referred to reference $[6]^{2}$.

For the calculations performed in this work, tracks of protons and carbon ions were simulated within a homogeneous liquid water cube of $20 \mu \mathrm{m}$ side length. The primary particles started in the centre of the volume at point $(0,0,0)$ and travelled $10 \mu \mathrm{m}$ along the $z$-direction.

For each track, the position with respect to the centre of the cubic volume and the amount of deposited energy was recorded for each individual ionisation of a water molecule. Those ionisations could be produced either by the projectile or by a secondary electron. The different energy deposits correspond to the five different ionisation shells of the water molecule used in the code: $10.79 \mathrm{eV}$, $13.39 \mathrm{eV}, 16.05 \mathrm{eV}, 32.3 \mathrm{eV}$ for the four valence levels and $539 \mathrm{eV}$ corresponding to the K-shell of the oxygen atom.

Simulations were performed for initially monoenergetic protons or carbon ions in a clinical relevant energy range: protons of energies $1 \mathrm{MeV}, 3 \mathrm{MeV}, 5 \mathrm{MeV}$, $10 \mathrm{MeV}, 25 \mathrm{MeV}, 50 \mathrm{MeV}$ and $100 \mathrm{MeV}$ and carbon ions of energies per nucleon of $10 \mathrm{MeV}, 40 \mathrm{MeV}, 70 \mathrm{MeV}$, $100 \mathrm{MeV}, 175 \mathrm{MeV}, 250 \mathrm{MeV}, 325 \mathrm{MeV}$ and $400 \mathrm{MeV}$ were used. In a second step we cut a track segment of $1 \mu \mathrm{m}$ length out of the full track starting $1 \mu \mathrm{m}$ behind the point where the simulation was started. We found that this procedure ensures that the considered track segment also contains energy depositions by secondary electrons created further upstream. The energy loss of the primary particles along the first $2 \mu \mathrm{m}$ path length remains below $6 \%$ of their initial energy for the energies and particles simulated, so that we can consider a constant energy for the projectile. Between 100 and 500 tracks per

\footnotetext{
${ }^{2}$ Geant4 DNA, www.geant4-dna.org. (Accessed: 2015-03-
}

energy were simulated which lead to at least 13000 energy transfer points within the considered track segment for the higher proton energies (50 MeV and $100 \mathrm{MeV}$ ) and a significantly higher number for all other radiation quantities. These tracks are the basis for the evaluations in the following sections. Indeed, for the analysis leading to the parametrisations, we used the position of the energy transfer point and the amount of energy deposited for all ionising interactions occurring in the simulated tracks.

\section{Track structure parametrisations}

We present three track structure parametrisations. The K-next neighbours parametrisation links the energy of the projectile to the ionisation pattern of the track using the distances to the 10 next neighbouring ionisations. A similar method to characterize track structure was already presented by reference [7]. The radial dose distribution is exploited for the second parametrisation which gives the amount of energy respectively dose which is delivered at a certain distance from the track core depending on the energy of the incident projectile. The third model concentrates on ionisation cluster size distributions and is based on the fact that a higher number of ionisations in a small volume results in a higher number of lesions on the DNA and an increased yield of double strand breaks $[8,9]$.

\subsection{K-next neighbours}

For the K-next neighbours parametrisation we made use of the Statistics Toolbox provided by MATLAB (The MathWorks, Inc. ${ }^{3}$ ) to characterise the ionisation patterns of the tracks by calculating the distances between K-next neighbours. Figure 1 gives the average euclidean distances from one energy transfer point to the next ten neighbouring transfer points for proton energies as indicated in the figure legend. In Figure 2 the same is shown for carbon ions. We calculated the distances to the next ten neighbouring ionisations for each ionisation point in all available tracks for the respective energy. Data was then divided into ten subgroups and we took the arithmetic mean value of the arithmetic mean values from each subgroup. The corresponding error is then given by the standard deviation of the mean values from the subgroups. Considering the average distances up to the tenth next neighbour of an ionisation ensures that the result is not dominated by the densely spaced ionisations in the track core. As one can see in Figures 1 and 2 the average distances to the next neighbours increase linearly with increasing order of the neighbour.

Therefore a linear function was fitted to the distances as a function of the neighbour:

$$
d=d_{0}+s(E) \cdot K
$$

where $d$ is the distance and $K$ the order of the neighbour. Data for each primary particle type and energy was fitted

\footnotetext{
3 www.mathworks.com
} 


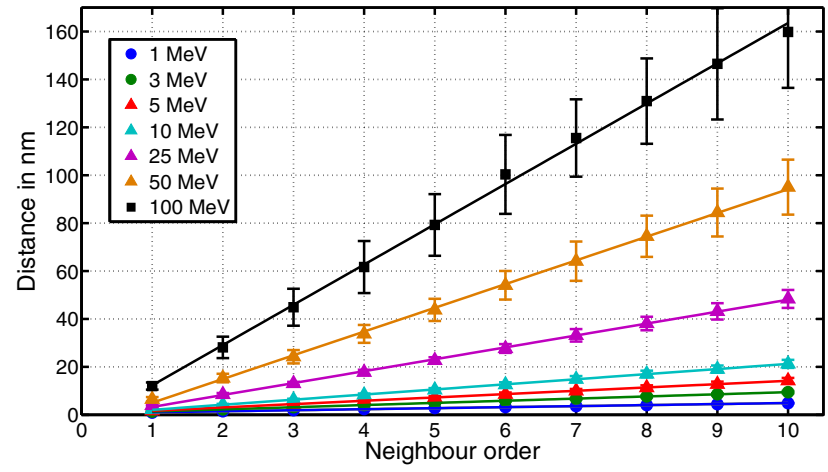

Fig. 1. Distances between K-next neighbour ionisation points for protons. Distances were determined for each ionisation in all available tracks for the particular energy. The results were then divided into ten subgroups. Each value shown is an average over the mean values from the 10 subgroups with the corresponding standard deviation. The lines are the linear fits to the data points.

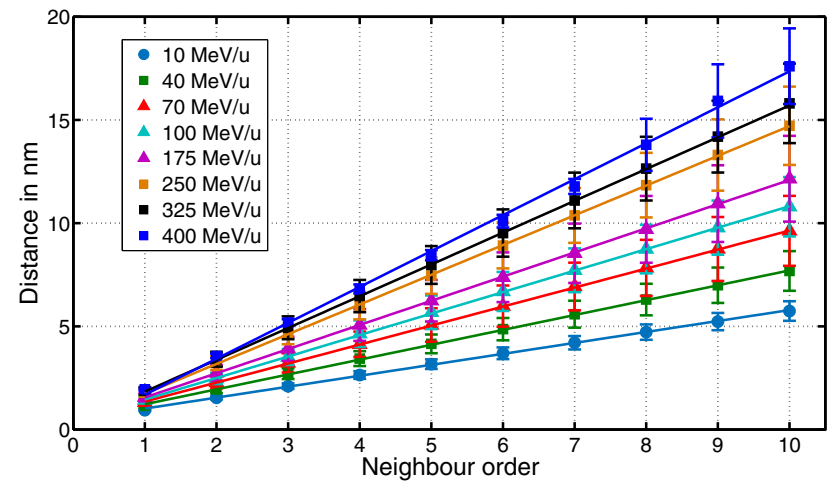

Fig. 2. Distances between K-next neighbour ionisation points for carbon ions. Distances were determined for each ionisation in all available tracks for the particular energy. The results were then divided into ten subgroups. Each value shown is an average over the mean values from the 10 subgroups with the corresponding standard deviation. The lines are the linear fits to the data points.

using equal weights for the data points. Figure 3 shows the obtained energy-dependent slope $s$ for protons plotted versus the energy of the incident projectile. Apparently the slope $s$ and energy $E$ follow a linear relationship as well:

$$
s(E)=c_{1} E+s_{0}
$$

with

$$
c_{1}=(0.18 \pm 0.02) \frac{\mathrm{nm}}{\mathrm{MeV}},
$$

and $s_{0}=(0.32 \pm 0.19) \mathrm{nm}$ with $95 \%$ confidence interval. For carbon ions the situation is slightly different. The slope increases with increasing projectile energy as well, but the relationship is best described by a power function (see Fig. 4):

$$
s(E)=a_{1}\left(\frac{E}{\mathrm{MeV}}\right)^{b_{1}}
$$

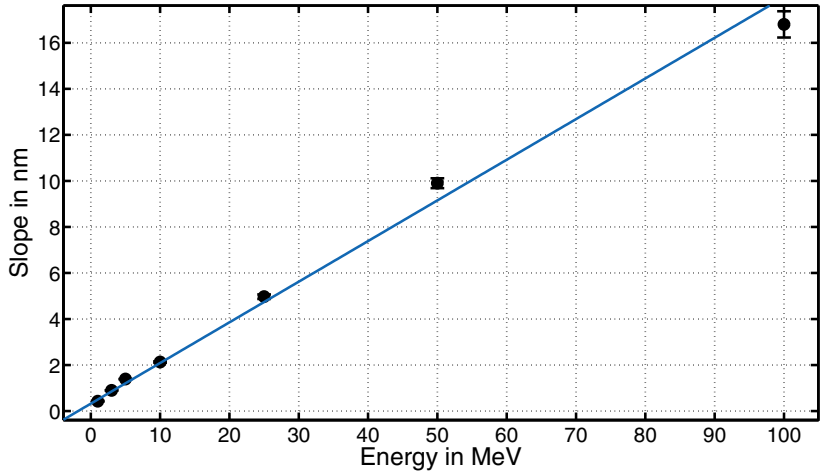

Fig. 3. The slope of the linear fits to the relation between order of neighbours and distances for all available proton energies. Errorbars show the $95 \%$ confidence intervals of the slope resulting from the linear regression. The solid line is the fit curve from a linear regression to the data points.

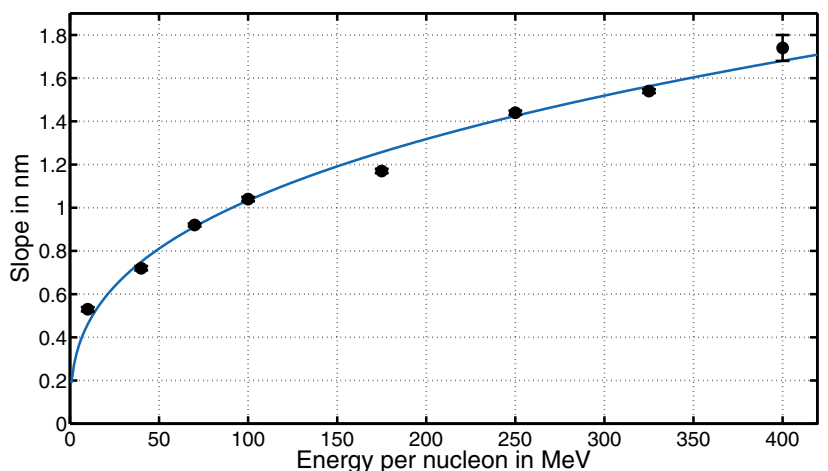

Fig. 4. The slope of the linear fits to the relation between order of neighbours and distances for all available carbon ion energies. Errorbars representing the $95 \%$ confidence intervals from the fits in Figure 2 are located within the marker symbol. The relation between energy of the incident projectile and the slope of the fit is best described by a power law.

with $a_{1}=(0.21 \pm 0.07) \mathrm{nm}$ and $b_{1}=(0.35 \pm 0.05)$. The comparison of the parametrisations for protons and carbon ions shows that the increase of the slopes for carbon ions is less steep with increasing energy. This is due to the fact that carbon ions tend to have more branches with close ionisations (independent from the track core) towards lower energies. Protons tend to produce ionisations that are more homogeneously distributed, which leads to a linear increase of the slope with energy.

\subsection{Radial dose distribution}

The second model describes the relation between the energy of the projectile and the amount of energy or dose which is delivered at a certain distance from the track core.

In the analysis, for each simulated track ionisation energies were summed up in concentric rings around the primary particle trajectory. The cylinder rings had a radial spacing of one nanometre. To obtain the absorbed dose, 


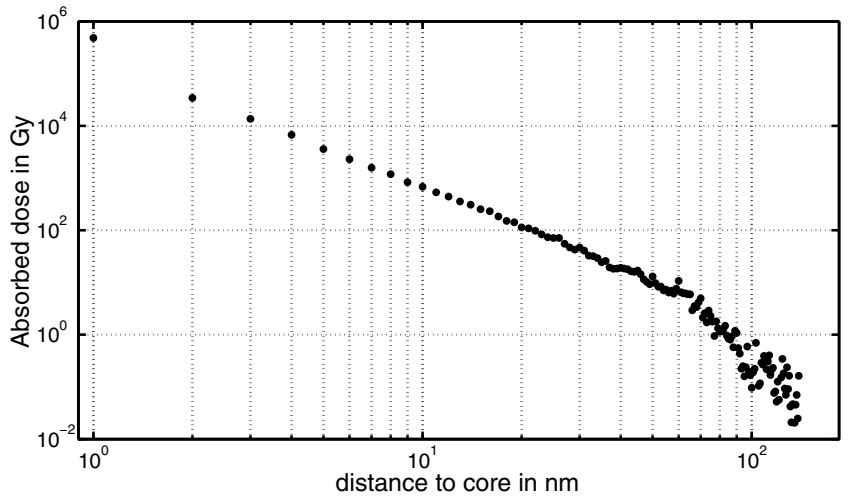

Fig. 5. Absorbed dose as a function of the distance to the track core for protons with an incident energy of $1 \mathrm{MeV}$. The result shown is averaged over 100 tracks. The spacing in the distance is $1 \mathrm{~nm}$.

the summed energy was divided by the product of water density, the area of the respective concentric ring and the length of the tracks. The following results were obtained by averaging over all available tracks.

Figure 5 shows the dose distribution in the track of $1 \mathrm{MeV}$ protons. The results agree with those from [10] who also analysed radial dose distributions using data produced with Geant4-DNA. In our analysis, the logarithm of the distance and the logarithm of the dose show a linear behaviour up to a distance of approximately $30 \mathrm{~nm}$ for protons and approximately $150 \mathrm{~nm}$ for carbon ions. This observation suggests that the relation of absorbed dose and radial distance from the track core is described by a power function.

Therefore we fitted the logarithm of our absorbed dose data as a function of the logarithm of the radial distance by polynomials of degree one for all available energies for protons and carbon ions. An example is shown in Figure 6 for $3 \mathrm{MeV}$ protons. The data points marked in red were excluded for the analysis. For larger distances this can be done without losing important information as the absorbed dose in these regions is relatively low and the aim of this parametrisation is to characterise the basic properties of the track structure. The first data point at $1 \mathrm{~nm}$ (representing all ionisations with $r \leq 1 \mathrm{~nm}$ ) was also excluded as it includes the projectile ionisations which can be easily reconstructed from the mean free path. Finally the absorbed dose $D$ depending on the radial distance from the track core $r$ can be calculated with the following power law:

$$
D(r)=a_{2}\left(\frac{r}{n m}\right)^{b_{2}} \times 1 \mathrm{~Gy}
$$

The data fitting was performed in two steps. In the first step, both parameters $a_{2}$ and $b_{2}$ were varied. From this first step, the mean value of the parameter $b_{2}$ was found to be -2.35 which is close to the well-known quadratic decrease of dose with distance [11]. The standard deviation of the different $b_{2}$ values was 0.1 . Therefore, in the second step of the fitting, $b_{2}$ was fixed at -2.35 and only parameter $a_{2}$ was allowed to vary.

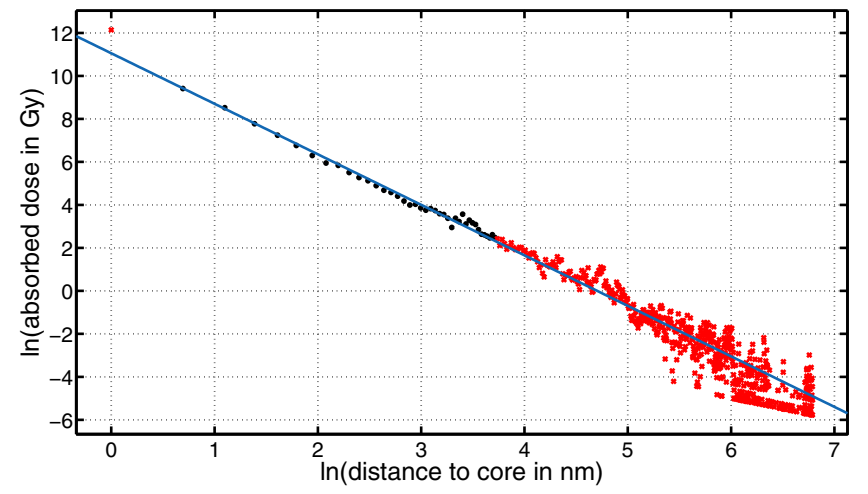

Fig. 6. Logarithm of the absorbed dose for protons with an incident energy of $3 \mathrm{MeV}$ as a function of the distance to the track core. The blue line is the linear fit for data points in black. The data points marked with red crosses were excluded for the fit.

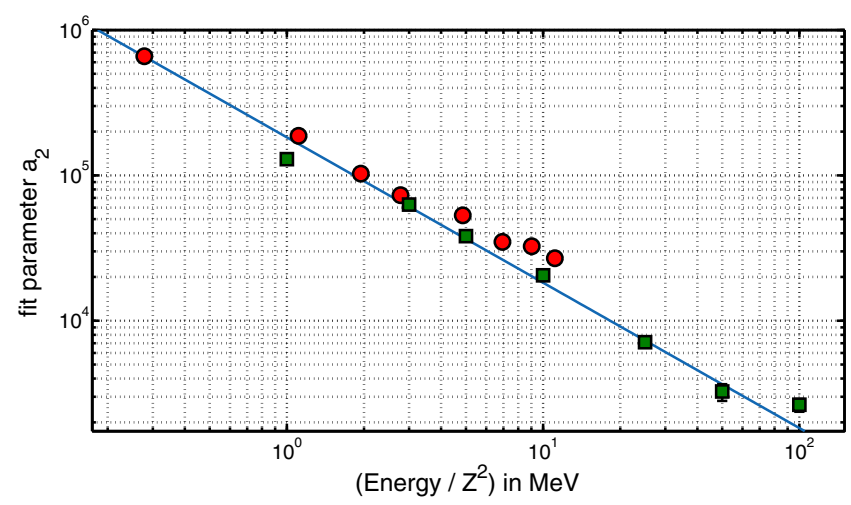

Fig. 7. Energy of the incident particle divided by the square of its charge number versus the fit parameter $a$. The errorbars represent the $95 \%$ confidence level of the fit to the logarithm of the absorbed dose. Data points for protons are represented by squares, carbon ions by circles. The blue line corresponds to the best-fit model curve $a_{2}(E)=\frac{k Z^{2}}{E}$ with $k=1.83 \times 10^{5} \mathrm{MeV}$.

In the final data fitting, the parameter which varies with particle energy and particle type is $a_{2}$. The squares in Figure 7 show the data obtained for the factor $a_{2}$ for protons of different energy while the circles show the values obtained for carbon ions. The $x$-axis of Figure 7 is the energy of the incident projectile divided by the square of its charge number. The data of both types of particles seem to follow the same relationship. The best fit could be achieved with the following hyperbolic function:

$$
a_{2}(E)=\frac{k Z^{2}}{E}
$$

with $k=(1.83 \pm 0.05) \times 10^{5} \mathrm{MeV}$ with $95 \%$ confidence interval. Using this formula the basic properties of the radial dose distribution can be calculated for protons and carbon ions for each desired energy in the clinical energy range. 


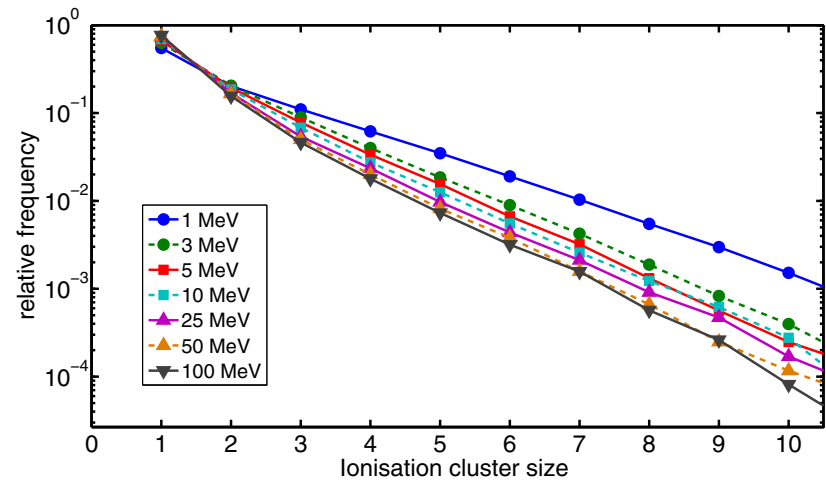

Fig. 8. Ionisation cluster size distributions of protons of different energies. On the $x$-axis the ionisation cluster size is shown while the $y$-axis gives the corresponding relative frequency. The probabilities for large cluster sizes are significantly higher for the $1 \mathrm{MeV}$ protons and decrease with increasing energy of the incident proton.

\subsection{Ionisation cluster size distributions}

The third model deals with ionisation cluster size distributions which describe the probabilities for ionisation clusters of a particular size to occur [12-16]. It is generally accepted that damage on subcellular structures - in particular the DNA - caused by ionisations or excitations is pivotal regarding the occurence of radiobiological effects $[17,18]$. Statistical parameters of ionisation cluster size distributions [19] can be used to correlate the spatial distribution of energy depositions with biological effects. Ionisation cluster size distributions were calculated for all available energies for protons and carbon ions by scoring the number of ionisations inside cylindrical target volumes. The size of the cylinders was chosen so that the volume resembles the geometry of a DNA segment which is a common practice in nanodosimetry $[9,20,21]$. The cylinders for this work have a height of $3.4 \mathrm{~nm}$ and a diameter of $2.3 \mathrm{~nm}$. 10 million cylinders per track were placed at randomly distributed sites within a cuboid which was determined by the outermost ionisations. A margin of $10 \mathrm{~nm}$ was added around this cuboid in order to avoid effects caused at the edge of the volume. The orientation of the cylinders was randomly chosen and cylinders could overlap. By counting the number of ionisations in each cylinder where an ionisation occured the conditional ionisation cluster size distributions could be determined. The relative frequency for each cluster size is shown for clusters up to a size of 10 in Figures 8 and 9. These frequencies are estimators for the conditional probability to obtain a particular ionisation cluster size given that at least an ionisation occurs. As can be seen the relative frequencies for cluster sizes are decreasing with increasing energy of the incident projectile.

Comparing the distributions for protons with those for carbon ions reveals that for the $10 \mathrm{MeV} / \mathrm{u}$ carbon ions the relative probabilities for cluster sizes between 2 and 10 do not decrease linearily in the semi-logarithmic representation as do the other distributions. The relative frequency for clusters sizes between 2 and 6 is equal and even lower

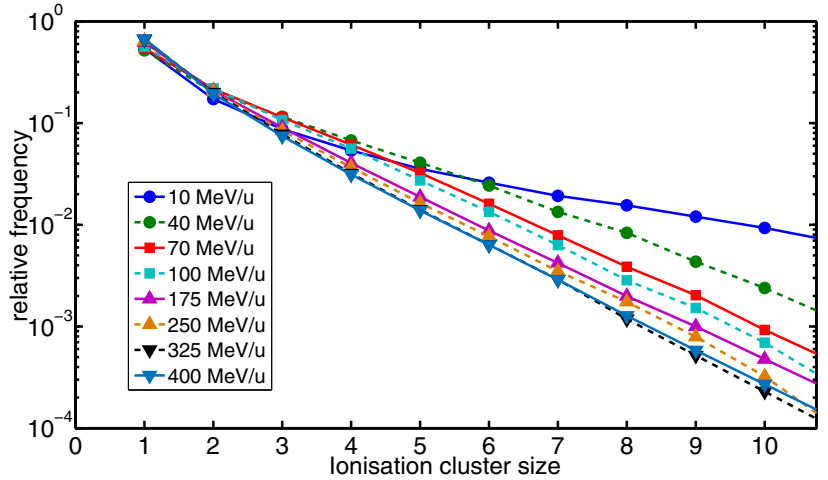

Fig. 9. Ionisation cluster size distributions for carbon ions of different energy per nucleon. On the $x$-axis the ionisation cluster size is shown while the $y$-axis gives the corresponding relative frequency. As for protons, carbon ions with lower energies show significantly higher frequencies for large cluster sizes.

compared to carbon ions with higher energies. The frequency for extremely large cluster sizes of seven ionisations or more in one cylinder is significantly higher. This might explain the enhanced biological effectiveness in tumor cell killing of carbon ions of energies close to the Bragg peak to some extent.

In order to gain an energy-dependent parametrisation of these distributions we calculated the first moment $M_{1}(Q)$ of the relative frequency distribution $P(\nu \mid Q)$ where $Q$ is the radiation quality and $\nu$ the cluster size. The mean ionisation cluster size can be calculated by summing up the products of the cluster size $\nu$ and the corresponding probability:

$$
M_{1}(Q)=\sum_{\nu=1}^{\infty} \nu P(\nu \mid Q)
$$

We calculated the mean ionisation cluster size for each projectile energy from the particular ionisation cluster size distributions and plotted $M_{1}$ as a function of the primary particle energy. The result for protons is shown in Figure 10. $M_{1}$ is decreasing with increasing energy. The same is shown for carbon ions in Figure 11. Both data sets can be fitted with a modified power law consisting of two terms:

$$
M_{1}(E)=a_{3}\left(\frac{E}{\mathrm{MeV}}\right)^{b_{3}}+c_{3}
$$

For protons the fit results in $a_{3}=0.67 \pm 0.04, b_{3}=$ $-0.58 \pm 0.08$ and $c_{3}=1.32 \pm 0.04$ while for carbon ions the parameters of the best fit are $a_{3}=3.64 \pm 1.77$, $b_{3}=-0.13 \pm 0.13$ and $c_{3}=-0.15 \pm 2.14$ with $95 \%$ confidence intervals. The blue lines in Figures 10 and 11 show the fit curves.

This parametrisation is able to predict the mean ionisation cluster size just from knowing the energy of the projectile. The same works for other properties of the distribution as well, for example the cumulative probability 


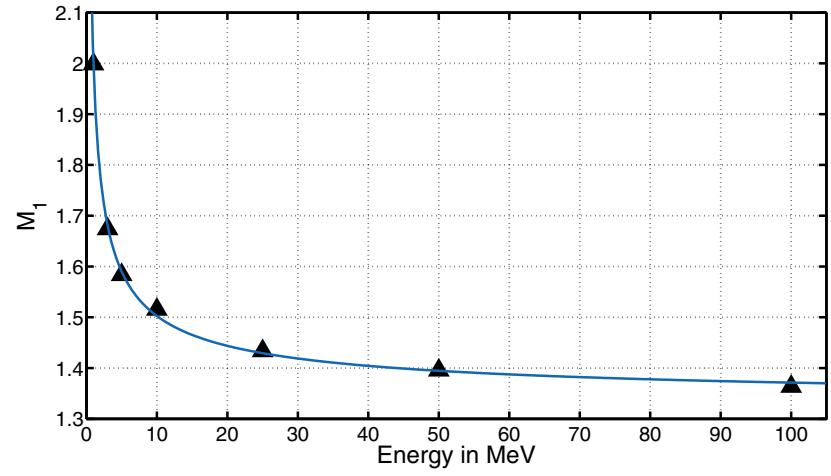

Fig. 10. Mean cluster size $M_{1}$ plotted against the energy of the incident proton. The blue line shows the best fit curve for a power function with two terms: $a_{3}\left(\frac{E}{\mathrm{MeV}}\right)^{b_{3}}+c_{3}$ where $a_{3}=0.67, b_{3}=-0.58$ and $c_{3}=1.32$.

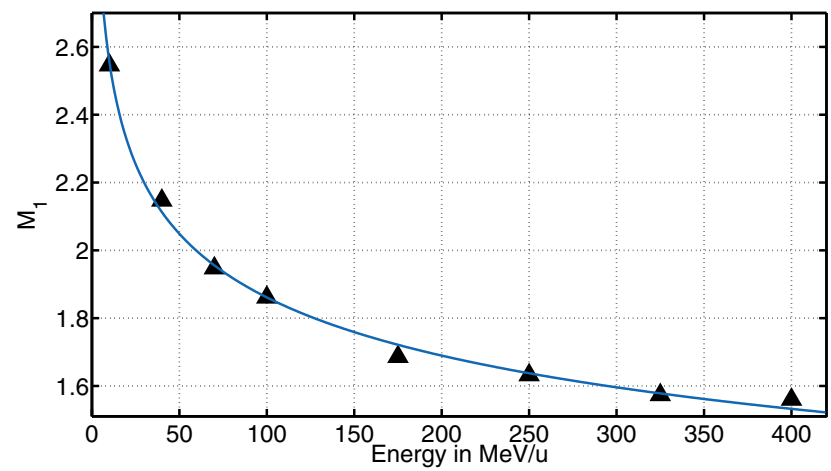

Fig. 11. Mean ionisation cluster size $M_{1}$ plotted against the energy of the incident carbon ion. The blue line shows the best fit curve for a modified power function with two terms: $a_{3}\left(\frac{E}{\mathrm{MeV}}\right)^{b_{3}}+c_{3}$ where $a_{3}=3.64, b_{3}=-0.13$ and $c_{3}=-0.15$.

for clusters with more than two ionisations

$$
F_{2}(Q)=\sum_{\nu=2}^{\infty} P(\nu \mid Q),
$$

or further combinations of moments of the distribution that seem to be promising measures regarding their potential to predict radiobiological effects [22].

\section{Conclusions}

We presented three track structure parametrisations. The first model can be used to easily derive the distances to the ten next neighbouring ionisations from the projectile energy. For each energy the slope of the distance depending on the order of the neighbour was determined. The fitting results show that this slope for protons is increasing linearly with proton energy while for carbon ions the slope increases with energy according to a power law. Going from high to low energies this means that the distances of the energy depositions are decreasing faster for carbon ions resulting in a higher probability for double strand breaks.
In the second model we described the radial dose distribution around the track core. The distribution can be characterised with a power law where the dose is decreasing with an exponential factor of -2.35 which is close to the well-known quadratic decrease of dose with distance described in the clinical used LEM model [11]. The second parameter which is the prefactor of the power law varies with projectile energy and was fitted with a hyperbolic function. The higher the energy the smaller is the factor and hence the dose absorbed around the track core. With this parametrisation one is able to determine the radial decrease of dose around the track core for protons and carbon ions in a clinical energy range with sufficient precision up to distances where a relevant amount of dose is absorbed.

The third model uses ionisation cluster size distributions which are widely used in the field of nanodosimetry to characterise track structure. Cluster size distributions have been calculated for protons and carbon ions in a clinical relevant energy range. The results show decreasing probabilities for larger clusters with increasing energy which is also reflected by the mean cluster size $M_{1}$. We fitted $M_{1}$ as a function of the projectile energy with a two-term powerlaw. The resulting functions for protons and carbon ions enable us to give a mean cluster size for each desired energy in the range between $1 \mathrm{MeV}$ and $100 \mathrm{MeV}$ for protons and $10 \mathrm{MeV} / \mathrm{u}$ and $400 \mathrm{MeV} / \mathrm{u}$ for carbon ions. With the help of such energy-dependent descriptions of nanodosimetric quantities like $M_{1}$, a basis is created for a fast calculation of track structure properties. The ionisation cluster size distributions and the associated statistical parameters are measurable quantities [23-27] and could in the future - when practical instruments have been developed - be used in the clinic to characterise track structure and radiation quality of the treatment beam. In combination with a model predicting the radiobiological outcome - for example the RMF model that is linking double-strand break induction to reproductive cell death $[28,29]$ - these parametrisations can be useful to achieve a higher accuracy for biological optimisation of treatment plans.

As mentioned above in Section 2 future versions of Geant4-DNA will probably describe the energy loss of carbon ions in more detail. As soon as more elaborate models become available, these simulations could easily be repeated to derive slightly modified parameters.

The presented track structure parametrisations provide a method to calculate essential track structure characteristics fast for each desired energy in the clinical energy range for protons and carbon ions. These models do not claim to give a detailed description of the track structure with nanometre resolution. Instead, they can quickly provide the crucial features of track structure for varying radiation quality.

In combination with adequate weighting methods (still to be developed) the parametrisations defined in this paper can be used to characterise the overall track structure of a mixed radiation field. Nuclear fragments in this radiation field can be taken into account with similar 
parametrisations that can be obtained with the presented method for all required particle species.

With an appropriate biological model added on top to predict the radiobiological outcome these parametrisations will be useful as an input for a treatment planning system based on track structure derived specifiers of local radiation quality. Using such parametrisations there will be no need to re-simulate track structure properties in each step of the iterative optimisation process with the objective to find the optimal treatment plan for each individual patient.

This work was carried out within the EMRP Joint Research Project BioQuaRT. The EMRP is jointly funded by the EMRP participating countries within EURAMET and the European Union.

\section{References}

1. D. Schulz-Ertner, H. Tsujii, J. Clin. Oncol. 25, 953 (2007)

2. H. Rabus et al., Eur. Phys. J. Web Conf. 77, 00021 (2014)

3. H. Palmans et al., British J. Radiol. 88, 20140392 (2015)

4. S. Agostinelli et al., Nucl. Instrum. Methods Phys. Res. A 506, 250 (2003)

5. S. Incerti et al., Int. J. Model., Simul. Sci. Comput. 01, $157(2010)$

6. S. Incerti et al., Med. Phys. 37, 4692 (2010)

7. G. Bäckström, M. Galassi, N. Tilly, A. Ahnesjö, J. Fernández-Varea, J. Med. Phys. 40, 064101 (2013)

8. R.W. Schulte et al., Zit. Med. Phys. 18, 286 (2008)

9. B. Grosswendt, Radiat. Environ. Biophys. 41, 103 (2002)
10. H. Wang, O.N. Vassiliev, Phys. Med. Biol. 59, 3657 (2014)

11. M. Scholz, A.M. Kellerer, W. Kraft-Weyrather, G. Kraft, Radiat. Environ. Biophys. 36, 59 (1997)

12. B. Grosswendt, Radiat. Protect. Dosimetry 110, 789 (2004)

13. B. Grosswendt, Radiat. Protect. Dosimetry 115, 1 (2005)

14. B. Grosswendt, Radiat. Protect. Dosimetry 122, 404 (2006)

15. G. Garty et al., Radiat. Protect. Dosimetry 122, 451 (2006)

16. G. Garty et al., Phys. Med. Biol. 55, 761 (2010)

17. D.T. Goodhead, Int. J. Rad. Biol. 65, 7 (1994)

18. P.L. Olive, Radiat. Res. 150, S42 (1998)

19. L.D. Nardo et al., Radiat. Environ. Biophys. 41, 235 (2002)

20. H. Nikjoo, D.T. Goodhead, Phys. Med. Biol. 36, 229 (1991)

21. P. Lazarakis et al., Phys. Med. Biol. 57, 1231 (2012)

22. B. Grosswendt, S. Pszona, A. Bantsar, Radiat. Protect. Dosimetry 126, 432 (2007)

23. A. Bantsar, B. Grosswendt, J. Kula, S. Pszona, Radiat. Protect. Dosimetry 110, 845 (2004)

24. V. Bashkirov et al., Radiat. Protect. Dosimetry 122, 415 (2006)

25. V. Conte, P. Colautti, B. Grosswendt, D. Moro, L. De Nardo, New J. Phys. 14, 093010 (2012)

26. G. Garty et al., Nucl. Instrum. Methods Phys. Res. A 492, 212 (2002)

27. G. Garty et al., Radiat. Protect. Dosimetry 99, 325 (2002)

28. D.J. Carlson, R.D. Stewart, V.A. Semenenko, G.A. Sandison, Radiat. Res. 169, 447 (2008)

29. M.C. Frese, V.K. Yu, R.D. Stewart, D.J. Carlson, Int. J. Radiat. Oncol., Biol., Phys. 83, 442 (2012) 